\title{
Real Time Stiffness Display Interface Device for Perception of Virtual Soft Object
}

\author{
Aiguo Song \\ Department of Instrument Science and Engineering \\ Southeast University \\ Nanjing 210096, P.R.China \\ a.g.song@seu.edu.cn
}

\author{
Dan Morris, J. Edward Colgate and Michael A. Peshkin \\ Department of Mechanical Engineering \\ Northwestern University \\ Evanston, IL 60208-3111, U.S.A \\ \{dmorris, Colgate,Peshkin\}@northwestern.edu
}

\begin{abstract}
A novel method based on deformable length of elastic element control (DLEEC) to realize the stiffness display for perception of virtual soft object is proposed, and the stiffness display interface device has been developed and is presented. The stiffness display interface device is composed of a thin elastic beam and an actuator to adjust the length of the beam. The deformation of the beam under a force is proportional to the third power of the beam length. By controlling the beam length, the stiffness display device can reproduce the stiffness of the virtual object from very soft to hard, so that the human fingertip can feel it as if he directly touches with the virtual object by interacting with the device. A real time position control algorithm is employed to guarantee the real time stiffness display.
\end{abstract}

Index Terms - haptic, virtual reality, stiffness display, teleoperation.

\section{INTRODUCTION}

In the field of virtual reality and teleoperation, haptic interaction between the human operator and a computer or telerobot plays an increasingly important role in performing delicate tasks, such as robotic telesurgery, virtual reality based training systems for telesurgery, virtual reality based rehabilitation systems [1][2][3], etc. These applications call for the implementation of effective means of haptic display to the human operator. Haptic display can be classified into two classes, one is haptic display in the tangent direction of the touched point, such as texture and friction display; another is haptic display in the normal direction, such as shape display and softness display. Previous research on haptic display mainly focused on texture display [4], friction display [5] and shape display [6]. Very little research dealt with softness display [7][8], which consists of stiffness display and compliance display. The stiffness information is important to the human operator for distinguishing among different objects when haptically telemanipulating or exploring the soft environment. Some effective haptic stiffness rendering methods for virtual reality have already been proposed, such as a finite-element based method [9], a precomputation based method [10], etc. An experimental system for measuring soft tissue phantom deformation during needle insertions has been developed and a method to quantify needle forces and soft tissue deformation is proposed in [11]. However, there are no effective stiffness display interface devices with a wide stiffness range from very soft to very hard for virtual reality yet. The existing PHANToM arm as well as some force feedback datagloves are inherently force display interface devices, which are unable to produce large stiffness display of hard object owing to the limitation of output force of the motors [12][13].

This paper presents a novel method to realize a real time stiffness display for human-computer interaction, which is based on deformable length of elastic element control (DLEEC). The proposed stiffness display interface device is composed of a thin elastic beam and an actuator to adjust the length of the beam. The deformation of the beam under a force is proportional to the third power of the beam length. By controlling the beam length, we can get the desirable stiffness, which tracks the stiffness of a virtual object or remote object with wide range from very soft to hard, to display to a fingertip of human operator. The remainder of the paper is organized as follows. In section II, the principle of the stiffness display based on DLEEC is discussed. In section III, PD controller based position control method for real time stiffness display is presented. The real time stiffness display interface device for perception of virtual soft object is described in section IV. Experiment results and conclusion are given in section $\mathrm{V}$ and VI, respectively.

\section{PRINCIPLE OF STIFFNESS DISPLAY BASED ON DLEEC}

The environment dynamics is usually expressed by a mass-spring-damp model as follows:

$$
f_{e}=m_{e} \ddot{x}_{e}+b_{e} \dot{x}_{e}+k_{e} x_{e}
$$

where $f_{e}$ is force acted on the environment, $x_{e}$ is displacement of the environment, and $m_{e}, b_{e}, k_{e}$ are mass, damp and stiffness of the environment, respectively. As to the soft environment discussed here, the displacement $x_{e}$ represents local deformation of its surface, and $m_{e}$ represents the local mass of its surface, which is relatively very small and usually can be omitted. If the damp is notable and the stiffness is small, the soft object is characterized by the compliance. If the reverse is the case, the soft object is characterized by the stiffness. In this paper, our research mainly focuses on the stiffness display, 
because for a lot of soft objects, such as most of the tissues of human body, stiffness is not only inherent, but also notable by comparison with damp or viscous. So that how to replicate the sense of stiffness to the user as if he directly touches with the virtual or remote soft environment is a primary issue in the softness display of the virtual environment and of the teleoperation.

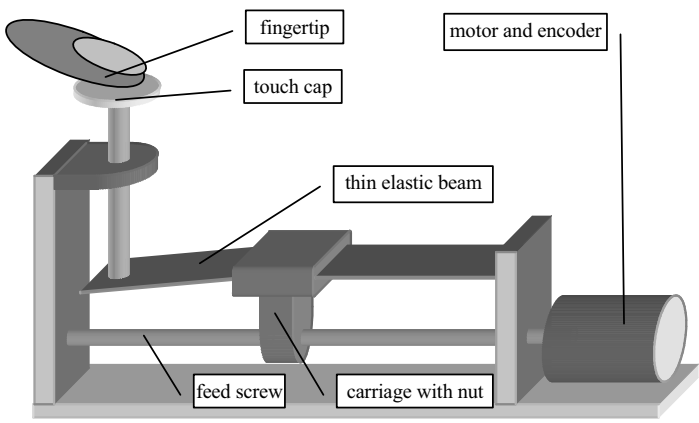

(a)

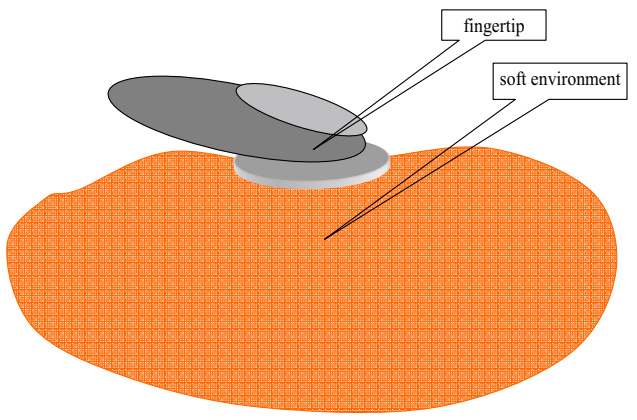

(b)

Fig. 1 Stiffness display of virtual soft environment

We design and fabricate a novel haptic display system based on DLEEC to realize the stiffness display of the virtual environment, which is shown in schematic form in Fig. 1(a). It consists of a thin elastic beam, feed screw, carriage with nut, and motor. The stiffness of the thin elastic beam is the function of deformable length of the beam $l$ seen in Fig 2. So the stiffness can be easily and smoothly changed to any value by controlling the deformable length of the thin beam $l$. Here, a motor, together with a feed screw and a nut, is used to control the position of the carriage, which determines the deformable length $l$.

In ideal case, when the human operator's fingertip pushes or squeezes the touch cap of the stiffness display interface device, he will feel as if he directly pushes or squeezes the soft environment with a small pad, seen in Fig. 1(b).

Fig. 2 shows the principle of the stiffness display based on DLEEC. Where, $y$ is vertical displacement of the end of the thin elastic beam when force $f$ acted on that point.

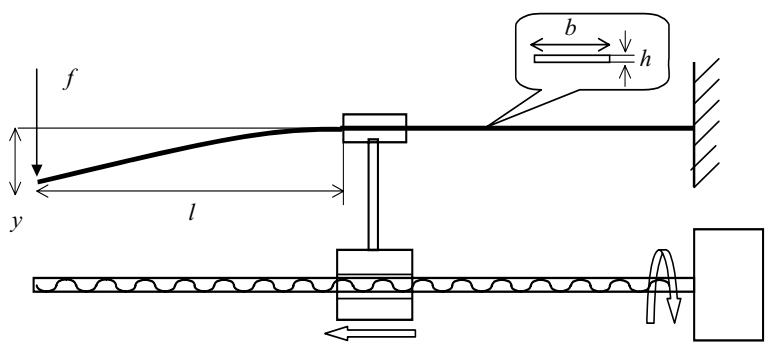

Fig. 2 Principle of the stiffness display based on DLEEC

According to the theory of mechanics of materials, the deformation of the thin elastic beam under the force $f$ can be given as:

$$
y=\frac{f l^{3}}{3 E I}
$$

where $E$ is Young's modulus, and $I$ is moment of inertia of the thin elastic beam.

$$
I=\frac{b h^{3}}{12}
$$

$b$ and $h$ are width and thickness of the thin elastic beam, respectively. Substituting (3) into (2) gives:

$$
y=\frac{4 f l^{3}}{E b h^{3}}
$$

Thus, the stiffness of the thin elastic beam, which is felt by the human fingertip at the touch cap of the device, can be expressed by an elastic coefficient as

$$
k=\frac{f}{y}=\frac{E b h^{3}}{4 l^{3}}=\rho \frac{1}{l^{3}}
$$

$$
\rho=\frac{E b h^{3}}{4} \text { is the gain of the stiffness. Equation }
$$

shows the stiffness at the free end of the cantilever $k$ is proportional to the third power of reciprocal of the deformable length $l$, which indicates that the stiffness $k$ can be changed with wide range as $l$ is changed.

Differentiating both sides of (5) with respect to time yields stiffness change ratio as:

$$
r_{k}=\frac{d k}{d t}=\frac{d k}{d l} \frac{d l}{d t}=-3 \rho \frac{1}{l^{4}} \times v_{\text {motor }}
$$

From the above formula, we know $r_{k}$ is proportional to the fourth power of reciprocal of the deformable length $l$, which indicates that the stiffness $k$ can be changed very quickly as $l$ is changed, especially when $l \rightarrow 0, r_{k} \rightarrow \infty$. Therefore the above formula means the ability of real time stiffness display based on DLEEC in our device.

\section{POSITION CONTROL FOR REAL TIME STIFFNESS DISPLAY}

Section II implies the core issue of the real time stiffness display actually is how to realize the real time position control of the carriage, which determines the deformable length $l$. Here, PD controller is employed for the real time position control. The control structure for the real time stiffness display is seen in Fig. 3. 


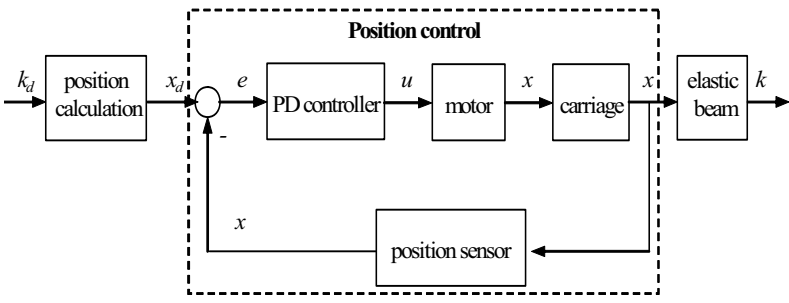

Fig. 3 Control structure for real time stiffness display

where $k_{d}$ is a destination stiffness to display, which comes from the virtual or remote soft environment. $x_{d}$ is a destination position of the carriage, which equals to the destination deformable length of the thin elastic beam $l_{d}$. Rewriting (5), we have:

$$
\begin{aligned}
& l_{d}=\sqrt[3]{\rho / k_{d}} \\
& x_{d}=l_{d}=\sqrt[3]{\rho / k_{d}}
\end{aligned}
$$

$\rho$ can be estimated by calibrating the stiffness change with respect to the deformable length of the thin elastic beam $l$. To simplify the estimation of $\rho$, let $z=1 / l^{3}$, and substitute it into (5), so that the power function in (5) can be transformed into a linear function as

$$
k=\rho \cdot z
$$

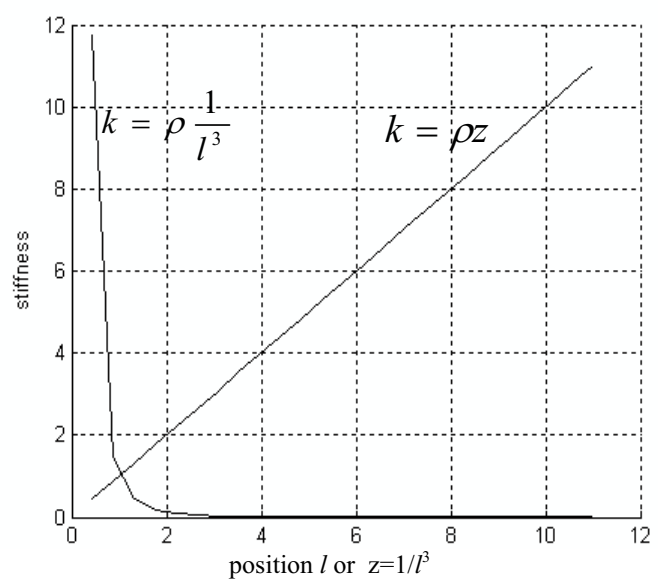

Fig. 4 Transform the power function into linear function

LMS method is used to estimate the parameter $\rho$ as follows

$$
\frac{\partial \sum_{i=1}^{n}\left|e_{i}\right|^{2}}{\partial \hat{\rho}}=0
$$

where $e_{i}$ is error of each measurement point.

$$
e_{i}=k_{i}-\hat{\rho} \cdot z_{i} \quad i=1, \cdots, n
$$

where $k_{i}$ is the $i$ th measurement value of stiffness at the $i$ th point $z_{i}$.

So that,

$$
\begin{gathered}
\sum_{i=1}^{n}\left(k_{i}-\hat{\rho} \cdot z_{i}\right) z_{i}=0 \\
\hat{\rho}=\frac{\sum_{i=1}^{n} k_{i} z_{i}}{\sum_{i=1}^{n} z_{i}{ }^{2}}
\end{gathered}
$$

The PD controller used here for position control of the carriage can be expressed as

$$
\begin{aligned}
& u=K_{p} e+K_{d} \frac{d e}{d t} \\
& e=x_{d}-x
\end{aligned}
$$

where $K_{p}$ is proportional control gain, $K_{d}$ is differential control gain, and $e$ is error between the destination position $x_{d}$ and the current real position $x$.

\section{REAL TIME STIFFNESS DISPLAY INTERFACE DEVICE}

The real time stiffness display interface device based on DLEEC method is shown in Fig. 5, which is composed of a thin elastic beam, a motor with an encoder, feed screw, carriage with nut, force sensor, position sensor, and a touch cap.

The material of the thin elastic beam in the stiffness display interface device is spring steel, whose Young's modulus of elasticity is $E=180 \times 10^{9} \mathrm{~N} / \mathrm{m}^{2}$. The size of the thin elastic beam is set as $60 \mathrm{~mm}$ long $\times 0.38 \mathrm{~mm}$ thick $\times 16.89 \mathrm{~mm}$ wide.

Substituting the above parameters into (5) can yield the minimum stiffness of the device:

$$
k_{\min }=0.305 \times 10^{3} \mathrm{~N} / \mathrm{m}=0.31 \mathrm{~N} / \mathrm{mm}
$$

$K_{\min }$ is the minimum stiffness of the softest object. Thus, the stiffness display range of the device is from 0.31 $\times 10^{3} \mathrm{~N} / \mathrm{m}$ to infinite, which almost covers the stiffness range of soft tissues in human body.

The position of the carriage is measured by an encoder with resolution of $8000 \mathrm{CPR}$. The displacement of the touch cap, which equals to the deformation of the end point of the thin elastic beam, is measured by a resistance based position sensor with $1 \%$ linearity. And the force acted by a fingertip on the touch cap is measured by a full bridge arrangement of resistance strain gauges with 0.025 lbs accuracy. The range of up-down movement of the touch cap when human fingertip jiggles it is from 0 to 2 $\mathrm{cm}$. 


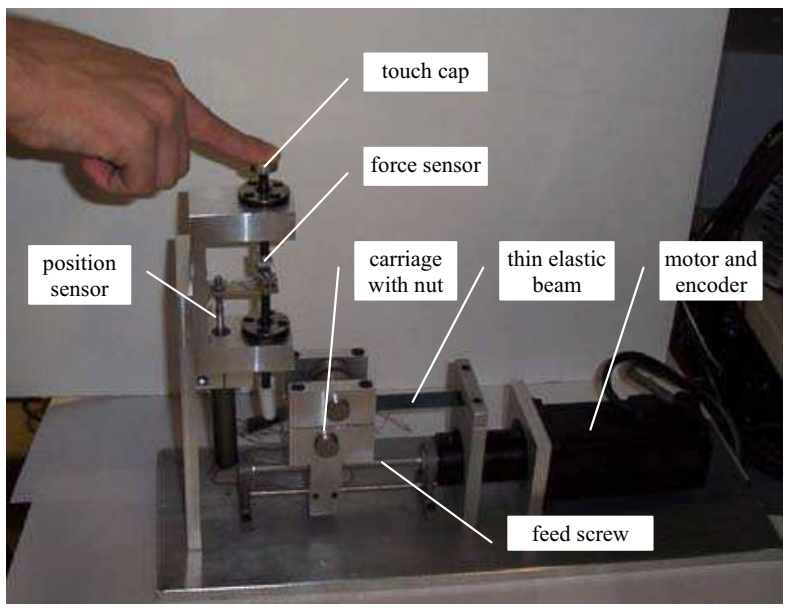

Fig. 5 Real time stiffness display interface device

\section{EXPERIMENT RESULTS}

The results of stiffness calibration of the stiffness display interface device are shown in Fig. 6. According to (12), the fitting curve of the relation between stiffness and $z=1 / l^{3}$ is shown in Fig. 7, and the $\hat{\rho}$ is estimated as

$$
\hat{\rho}=1.15 \times 10^{4}\left(N \cdot \mathrm{mm}^{2}\right)
$$

The Fig. 6 and Fig. 7 demonstrate the validity of the equation (5), although there exists some difference between experimental curve and fitting curve. The difference mainly comes from the effect of friction between the cantilever beam and the carriage, and from the effect of nonlinear property when the length of the cantilever beam becomes small and the ratio of end point deformation to the length of the cantilever beam becomes large.

In order to overcome the bad effects of friction and nonlinear property so as to control the deformable length of the thin elastic beam precisely, we make a table to record the relationship between the stiffness and the deformable length of the beam point by point based on calibration data. And a table looking-up method is used for transforming a destination stiffness to a destination length of the cantilever beam.

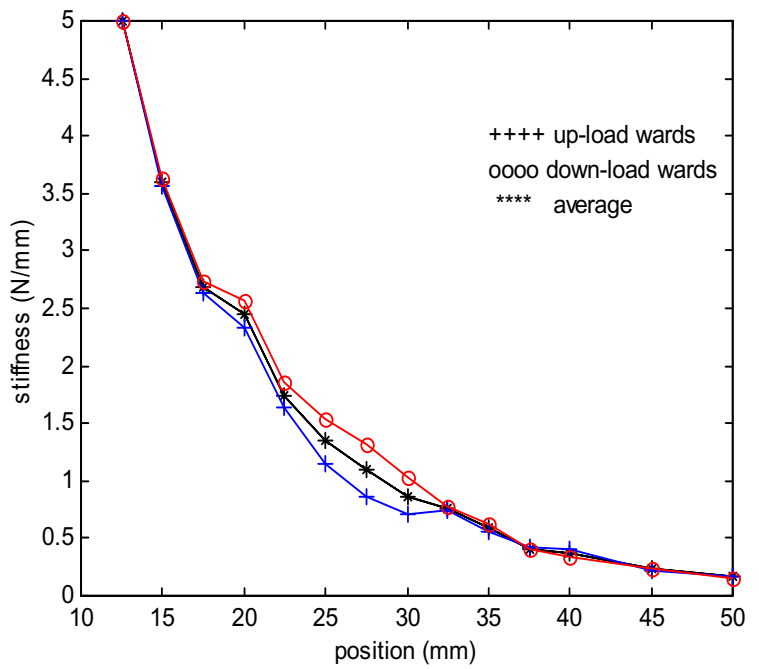

Fig. 6 Results of stiffness calibration

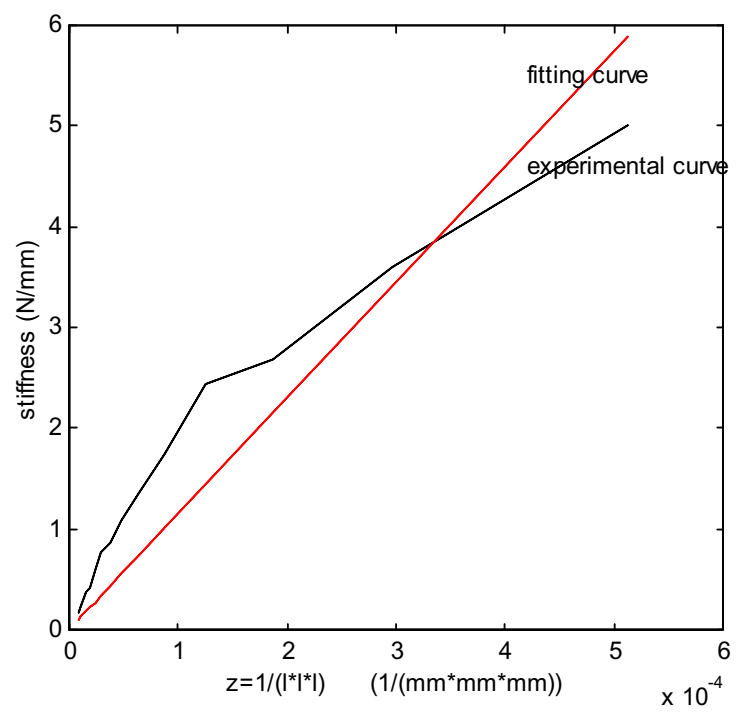

Fig. 7 Fitting curve of characteristic of stiffness

The result of the position control of the carriage is shown in Fig. 8. Here, the proportional control gain and the differential control gain of the PD controller are set as

$$
\begin{gathered}
K_{p}=5 \times 10^{-3} \\
K_{d}=1.6 \times 10^{-4}
\end{gathered}
$$

The above setting is based on experience and some experiment results.

Fig. 8 implies the control of deformable length of the thin elastic beam is real time control.

The trajectory of stiffness display which tracks the destination stiffness change of a virtual soft object is shown in Fig. 9. Note that the destination stiffness is set as step square pulses, which corresponds to the typical change of stiffness of some soft tissues with blood vessels beneath the surface.

The stiffness display experiment results demonstrate that the stiffness display interface device is able to 
replicate the stiffness of the virtual soft object quickly and accurately.

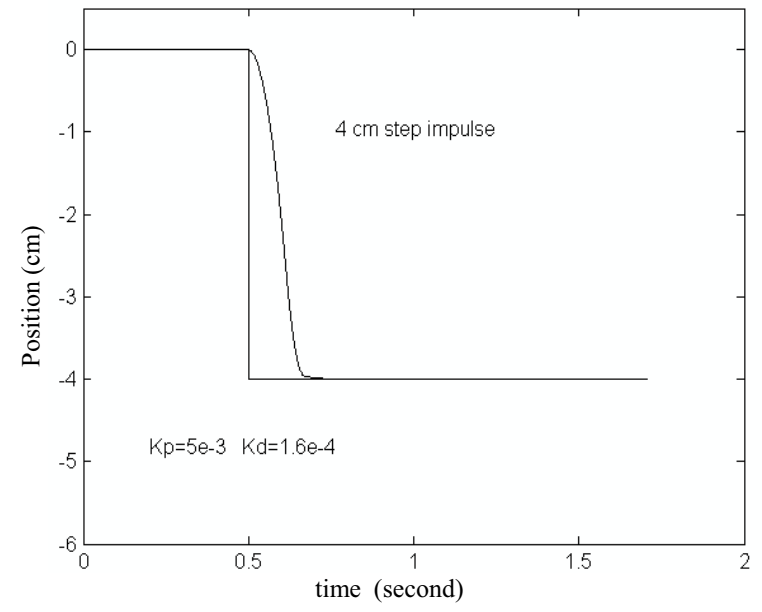

Fig. 8 Position control result

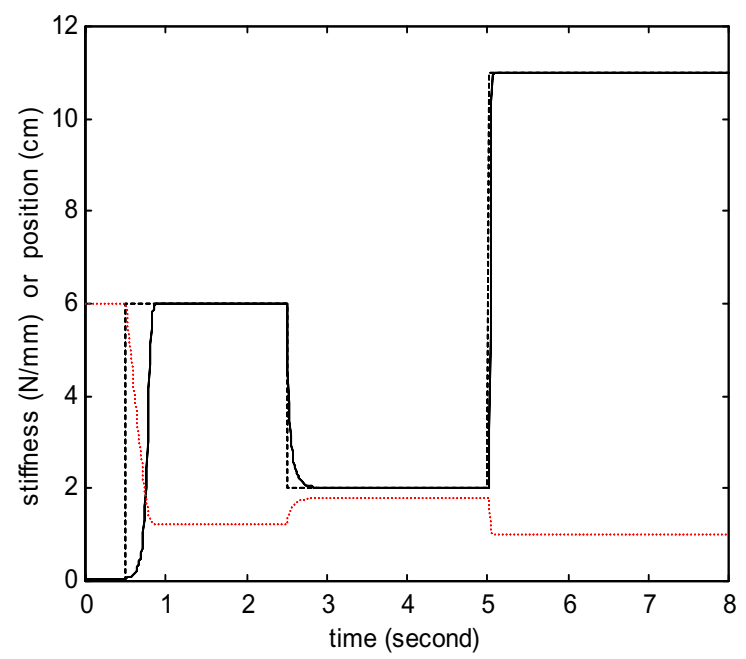

The solid line represents displayed stiffness, the dashed line represents destination stiffness, and the dotted line represents position of the carriage controlled by PD controller.

Fig. 9 Stiffness display experiment results

\section{CONCLUSION}

A novel method based on deformable length of elastic element control (DLEEC) to realize the stiffness display for virtual or remote soft object is proposed. The proposed stiffness display interface device is composed of a thin elastic beam and an actuator to adjust the length of the beam. The deformation of the beam under a force is proportional to the third power of the beam length. By controlling the beam length, we can get the desirable stiffness quickly. And the PD controller based control structure for stiffness display is given. The experimental results demonstrate the stiffness display interface device is able to realize the real time stiffness display for perception of virtual object.
Aiguo Song would like to thank 973 Program of China (2002CB312102) and Hwa-Ying Culture and Education Fund for their offering of fellowship and academic holiday to him as a visiting scientist at Northwestern University. Thanks to Prof. Kevin Lynch, Mr. Mark Salada and Mr. Jim Lipsey for their good advice. And this paper is also partly supported by the Huo Yingdong Education Fund for Young Teacher and Natural Science Foundation of China (60475034).

\section{REFERENCES}

[1] Paolo Dario, Blake Hannaford, Ariama Menciassi, "Smart surgical tools and augmenting devices," IEEE Trans. on Robotics and Automation, vol.19, no.5, 2003, pp.782-792

[2] Russel H. Taylor, Dan Stoianovici, "Medical robot in computerintegrated surgery," IEEE Trans. on Robotics and Automation, vol.19, no.5, 2003, pp.765-781

[3] Viorel G. Popescu, Grigore C. Burdea, Mourad Bouzit, Vincent R. Hentz, "A virtual-reality-based tele-rehabilitation system with force feedback," IEEE Trans. on Information Technology in Biomedicine, vol.4, no.1, 2000, pp.45-51

[4] Lkei Y., Yamada M., Fukuda S., "A new design of haptic texture display - texture display2 - and its preliminary evaluation," Proceedings of Virtual Reality Annual International Symposium, 2001, pp.21-28

[5] Richard C., Cutkosky Mark R., "Friction modeling and display in haptic applications involving user performance," Proceedings of IEEE International Conference on Robotics and Automation, 2002, pp.605-611

[6] Kammermeier P., Buss M.; Schmidt G, ".Dynamic display of distributed tactile shape information by a prototypical actuator array," Proceedings of IEEE International Conference on Intelligent Robots and Systems, vol. 2, 2000, pp.1119-1124

[7] Antonio Bicchi, Enzo P. Scilingo, Danilo De Rossi, "Haptic discrimination of softness in teleoperation: the role of the contact area spread rate," IEEE Trans. on Robotics and Automation, vol.16, no.5, 2000, pp.496-504

[8] Moy G., Wagner C.; Fearing R.S., "Compliant tactile display for teletaction," Proceedings of IEEE International Conference on Robotics and Automation, vol.4, 2000, pp.3409-3415

[9] Shahram Payandeh, Naoufel Azouz, "Finite elements, mass-springdamper systems and haptic rendering," Proceedings of 2001 IEEE International Symposium on Computational Intelligence in Robotics and Automation, 2001, pp.224-229

[10]Doug L. James, Dinesh K. Pai, “A unified treatment of elastostatic contact simulation for real time haptics," Haptics-e, vol.2, no.1, 2001 (http://www.haptics-e.org)

[11]Simon P. Dimaio, S.E.Salcudean, "Needle insertion modeling and simulation," IEEE Trans. on Robotics and Automation, vol.19, no.5, 2003, pp.864-875

[12]T.Massie, K.Salisbury, "The PHANToM haptic interface: a device for probing virtual objects," Proceedings of ASME WAM, DSCvol.55-1, 1994, pp.295-300

[13]Grigore C. Burdea, "Haptics issues in virtual environments," Proceedings of Computer Graphics International Conference, CGI, 2000, pp.295-302 